\title{
ACM and IEEE Contribution to Curriculum Change in Computer Science
}

\author{
Sónia Rolland Sobral \\ REMIT, Universidade Portucalense (PORTUGAL) \\ doi: $\underline{10.21125 / \text { inted.2020.2160 }}$
}

The first curriculum studies for undergraduate studies in Computer Science appeared in March 1968, when the Association for Computing Machinery (ACM) published an innovative and necessary document, Curriculum 68: Recommendations for academic programs in computer science. Since then, efforts have been made to make curriculum recommendations for universities around the world. In 1991, IEEE (Institute of Electrical and Electronics Engineers) and ACM joined to publish a new document, computing curricula 1991: report of the ACM/IEEE-CS Joint Curriculum Task Force. In 2005, with the Computing Curriculum 2005: The Overview Report, there was made a distinction between Computer Engineering (CE), Computer Science (CS), Information Systems (IS), Information Technology (IT), and Software Engineering (SE). In this way, the new curriculum reports have come to focus on a specific area, and there is a departure from the core and elective areas by each of the specific cases.

The importance of ACM and IEEE in curriculum design has been enormous for those who have the responsibility of designing and redesigning curricula in the area of computer science. These curriculum recommendations point out a number of different paths, namely in relation to the already mentioned distinction between areas but also the distinction that is made by university size, previous knowledge of the students, and also duration of studies, among other variables.

Computing is not watertight and computer science education reveals those changes. The world has changed a lot in the last 50 years of curriculum recommendations. This article provides a historical review of the different ACM documents and the task-force ACMIEEE recommendations while reviewing what has happened in the world for these changes to occur. The computer world, especially those linked to university computer science education, should be grateful to ACM and IEEE for having made efforts to update and improve computer education, keeping up with the changes that have taken place in the world during the last 50 years.

keywords: curriculum recommendations, acm, ieee. 